\title{
Subjective and Objective Quality Evaluation Study of BPL-PLC Wired Medium
}

\author{
Grzegorz Debita ${ }^{1, *}$, Przemyslaw Falkowski-Gilski² ${ }^{2}$ Marcin Habrych ${ }^{3}$, Bogdan Miedzinski ${ }^{3}$, \\ Bartosz Polnik ${ }^{4}$, Jan Wandzio ${ }^{5}$, Przemyslaw Jedlikowski ${ }^{6}$ \\ ${ }^{1}$ General Tadeusz Kosciuszko Military University of Land Forces, \\ Czajkowskiego St. 109, 51-147 Wroclaw, Poland \\ ${ }^{2}$ Faculty of Electronics, Telecommunications and Informatics, Gdansk University of Technology, \\ Narutowicza St. 11/12, 80-233 Gdansk, Poland \\ ${ }^{3}$ Faculty of Electrical Engineering, Wroclaw University of Science and Technology, \\ Wybrzeze Wyspianskiego St. 27, 50-370 Wroclaw, Poland \\ ${ }^{4}$ KOMAG Institute of Mining Technology, \\ Pszczynska St. 37, 44-101 Gliwice, Poland \\ ${ }^{5}$ KGHM Polska Miedz S. A., \\ Sklodowskiej-Curie St. 48, 59-301 Lubin, Poland \\ ${ }^{6}$ Faculty of Electronics, Wroclaw University of Science and Technology, \\ Wybrzeze Wyspianskiego St. 27, 50-370 Wroclaw, Poland \\ grzegorz.debita@awl.edu.pl
}

\begin{abstract}
This paper presents results of research on the effectiveness of bi-directional voice transmission in a $6 \mathrm{kV}$ mine cable network using BPL-PLC (Broadband over Power Line Power Line Communication) technology. It concerns both emergency cable state (supply outage with cable shorted at both ends) and loaded with distorted current waveforms. The narrowband (0.5 MHz-15 MHz) and broadband (two different modes, frequency range of $3 \mathrm{MHz}-7.5 \mathrm{MHz}$ ) transmission efficiency, considered as an objective study, was correlated with the level of noise in the cable for both induction-inductive and mixed capacitive-inductive coupling of the measurement devices and/or modems. The quality of voice transmission, considered as a subjective study, was assessed by a group of 15 people aged between 25 and 35 years old. The test audio signals included samples in 3 languages sourced from ITU (International Telecommunication Union) and processed in 3 bitrates ranging from $8 \mathrm{kbps}$ to $24 \mathrm{kbps}$.
\end{abstract}

Index Terms-BPL communication system; MV cable network; Quality evaluation; Signal processing; Voice transmission.

\section{INTRODUCTION}

The BPL-PLC (Broadband over Power - Power Line Communication) technology is an alternative to other methods used in signal transmission. The main advantage is its low application cost, since it enables to utilize existing low and/or high voltage network infrastructure without extra installation expenditures and operator fees. However, one has to note that this technology, in general, is sensitive to environmental electromagnetic compatibility (EMC) interference, both conducted and induced. Therefore, appropriate measures must be taken into account [1]-[6].

Manuscript received 25 January, 2020; accepted 16 May, 2020.
Authors, experienced in the mining industry, have found that application of the BPL-PLC (with frequency range of $3 \mathrm{MHz}-32 \mathrm{MHz}$ ) in mine medium voltage (MV) networks can be beneficial [7]-[9]. It can be used not only to, e.g., control environmental conditions, but most of all as a voice/image transmission medium, including the so-called last chance, in case of threats and mine disasters. In fact, MV cable networks are theoretically the most resistant to mechanical damage. Therefore, an effective signal transmission, especially voice commands, can be maintained even under permanent damage of phase conductors, along with battery powered critical devices.

It should be also emphasized that continuity of voice communication, using BPL-PLC, is likewise important to provide in municipal power cable networks, especially under hazards, e.g., black-outs, as well as any other potential military threats [7]. However, in order to meet necessary requirements and overcome encountered problems, when talking about high-efficiency BPL-PLC transmission, including voice communication, the impact of both external and internal threats must be recognized. It also requires appropriate matching of the transmitting and receiving devices to the characteristic impedance of the utilized medium voltage cable, as well as type of effective cable coupling [9].

Furthermore, sufficiently high bitrate in order to obtain appropriate quality of the speech signal itself is also needed. Currently, commercial broadcasting and/or streaming services require a bitrate of $48 \mathrm{kbps}-64 \mathrm{kbps}$ [10]-[13]. Such high bitrates may be sometimes difficult to obtain. Moreover, objective high-quality BPL-PLC signal transmission, e.g., relatively high SNR values, is not necessarily synonymous with high-quality subjective 
feelings.

This paper discusses test results of signal transmission efficiency correlated with the efficiency of bi-directional voice transmission in a $6 \mathrm{kV}$ mine cable network utilizing BPL-PLC technology. They concern both investigations under simulated emergency conditions (cable line deenergized and shorted at both ends) and a real line loaded with distorted current waveforms. The measurements, both narrowband $(0.5 \mathrm{MHz}-15 \mathrm{MHz})$ and broadband (two different modes, frequency range of $3 \mathrm{MHz}-7.5 \mathrm{MHz}$ ), were correlated with noise level values in the cable itself.

Tests were performed for both induction-inductive and capacitive-inductive coupling with measuring devices and/or BPL-PLC cable modems. The quality of transmitted voice messages was later assessed by a group of 15 people (25-30 years old). The utilized audio samples, in 3 different languages, were sourced from ITU (International Telecommunication Union) [14]. The degraded (transmitted) samples were processed in 3 different bitrates, namely: $8 \mathrm{kbps}, 16 \mathrm{kbps}$, and $24 \mathrm{kbps}$. Based on obtained results, appropriate practical conclusions have been formulated.

\section{OBJECTIVE EVALUATION TESTS}

Tests regarding signal transmission efficiency, in the medium voltage mining cable, were carried out under realtime (mining) operating conditions taking into account both noise level in the cable and its loading with distorted current waveforms. The transmission quality for emergency cable conditions, e.g., disconnected power supply shorted and grounded at both ends, was checked. The effect of powering the cable on non-linear loads, being the source of higher harmonics of both current and voltage, was also examined.

In order to select the frequency (modes) used in BPL-PLC with two-way voice transmission, appropriate narrowband investigations were first carried out. They were performed for both induction-inductive and capacitive-inductive coupling of the measuring devices and/or cable modems. The frequency of the narrowband transmission was changed from $0.5 \mathrm{MHz}$ to $15 \mathrm{MHz}$, respectively. A fragment of a $6 \mathrm{kV}$ cable line, with a length of about $300 \mathrm{~m}$, was selected for the test [8]. It was located in the sidewalk of the mine shaft; the ambient temperature was equal to approximately $12{ }^{\circ} \mathrm{C}$.

\section{A. Noise Measurements}

The method of measuring the value and waveform level (in terms of EMC disturbances between $0.5 \mathrm{MHz}-32 \mathrm{MHz}$ ) in the cable under non-linear load $(6 \mathrm{kV}, 80 \mathrm{~A})$ is shown in Fig. 1. Tests were carried out for both capacitive and inductive coupler installed at cable supply point $\mathrm{A}$. In this case, a spectrum analyzer, namely, USB-SA44B (resolution bandwidth less than $1 \mathrm{~Hz}$ ) was used as the measuring instrument [15].

During the test, the cable supplied non-linear mining loads (motors with frequency converters). The effective value of distorted current was equal to approximately $84 \mathrm{~A}$. The harmonic content $\left(3^{\text {rd }}, 5^{\text {th }}, 7^{\text {th }}\right.$, and $\left.11^{\text {th }}\right)$ in voltage was relatively small, so that the total harmonic distortion in voltage (THDu) value was within $0.5 \%-2.7 \%$. However, in current, the value of the resultant THDi factor was slightly higher, but did not exceed $9 \%$.

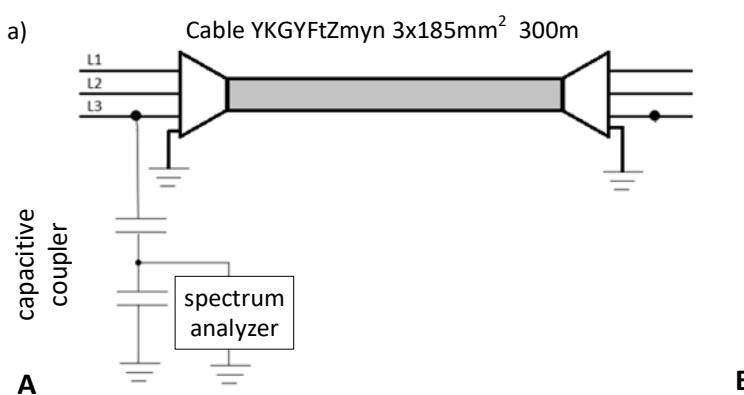

b)

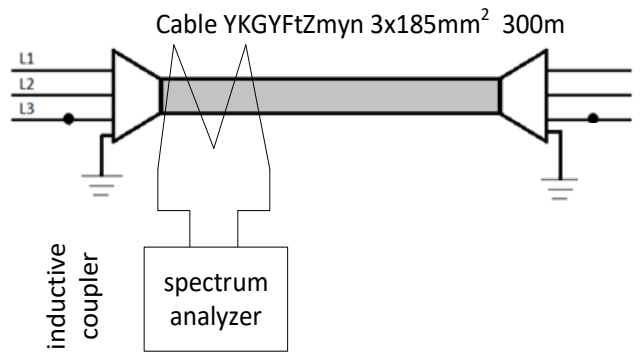

A

Fig. 1. Noise measurement in a $6 \mathrm{kV}$ cable under load $(84 \mathrm{~A})$ : a) capacitive coupling of the spectrum analyzer with cable at point $\mathrm{A}, \mathrm{b}$ ) inductive coupling of the spectrum analyzer with cable at point $\mathrm{A}$.

This fact is illustrated by the measured courses of this coefficient (for 3 phases), shown in Fig. 2.

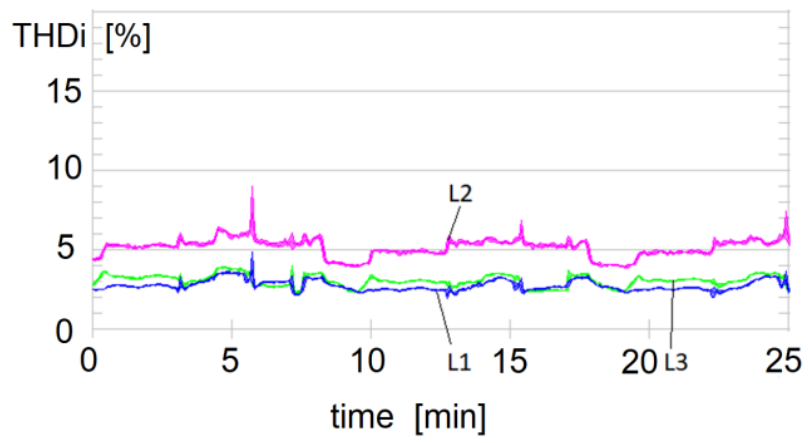

Fig. 2. Measured values of resultant THDi (harmonics: $3^{\text {rd }}, 5^{\text {th }}, 7^{\text {th }}, 9^{\text {th }}$, and $\left.11^{\text {th }}\right)$ in 3-phases during $6 \mathrm{kV}$ cable operations.

The test carried out for both types of coupling showed that the maximum noise value did not exceed $-50 \mathrm{dBm}$. Whereas, for the capacitive coupler, the value practically did not change with frequency as shown in Fig. 3.

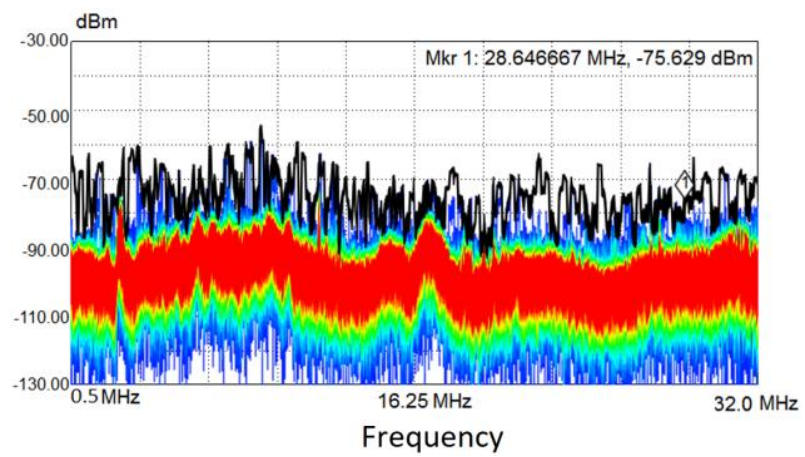

Fig. 3. Noise spectrum $(0.5 \mathrm{MHz}-32 \mathrm{MHz})$ in a $6 \mathrm{kV}$ cable under nonlinear load (THDi $<9 \%$ ) - capacitive coupler.

In case of the inductive coupler, as shown in Fig. 4, the 
noise value slightly decreases with frequency, especially around the average value. When comparing measurements of unloaded and shorted cable, and for cable loaded with distorted currents, it can be stated that the distortions of current and voltage waveforms (low harmonics $>3^{\text {rd }}$ ) have no visible effect on the value of noise.

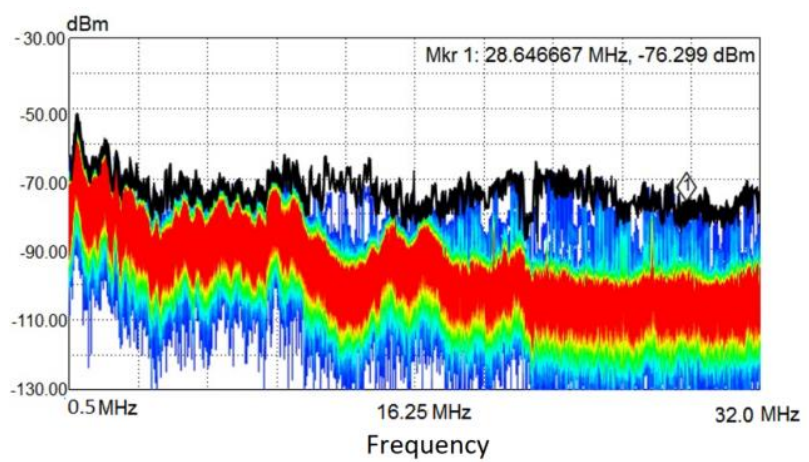

Fig. 4. Noise spectrum $(0.5 \mathrm{MHz}-32 \mathrm{MHz})$ in a $6 \mathrm{kV}$ cable under nonlinear load (THDi $<9 \%$ ) - inductive coupler.

This fact is important when considering the efficiency of the BPL-PLC technology. However, the load on the cable seems to affect the noise level to some extent. This may be the result of the impact of the magnetic field of the load current on the magnetic permeability of ferromagnetic materials, e.g., cable armor.

\section{B. Narrowband Transmission}

The configuration for the narrowband transmission efficiency test is shown in Fig. 5. It concerned both induction-inductive and mixed capacitive-inductive coupling of the measuring apparatus with a $6 \mathrm{kV}$ cable under load.

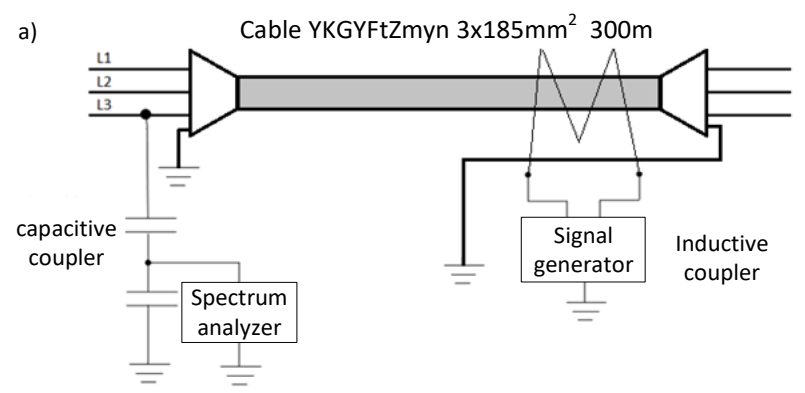

A

b)

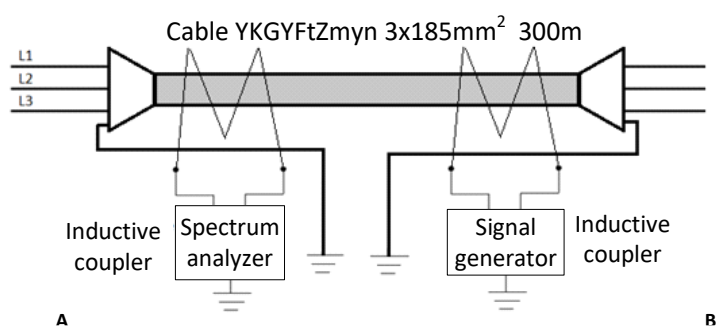

Fig. 5. Efficiency measurements of narrowband transmission in a $6 \mathrm{kV}$ cable under load: a) capacitive-inductive coupling, b) induction-inductive coupling.

The input signal frequency (adjustable in the range of $0.5 \mathrm{MHz}-15 \mathrm{MHz}$ range) with a constant amplitude of $10 \mathrm{dBm}$ was injected from a generator, namely, AFG-2225 [16] installed at point $B$. The value of the output signal was recorded using the USB-SA44B spectrum analyzer [15] located at the opposite end of the cable at point A. Obtained measurements showed that the best transmission efficiency (from $-22 \mathrm{dBm}$ to approximately $-38 \mathrm{dBm}$ ) for the inductioninductive coupling occurred within the frequency range from approximately $2 \mathrm{MHz}$ to $8 \mathrm{MHz}$. Whereas, for the capacitive-inductive coupling (from $-40 \mathrm{dBm}$ to approximately $-54 \mathrm{dBm}$ ), it had a quite similar range from approximately $2.5 \mathrm{MHz}$ to $7.5 \mathrm{MHz}$. An example of the measured relationship is shown in Fig. 6.

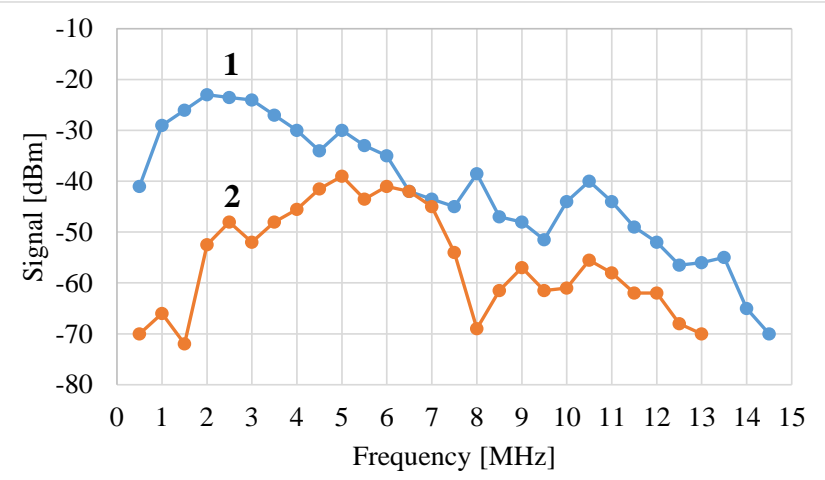

Fig. 6. Output signal gain depending on the frequency of the input signal (constant amplitude equal to $10 \mathrm{dBm}$ ) for narrowband transmission in the analyzed $6 \mathrm{kV}$ loaded cable: (1) induction-inductive coupling, (2) capacitive-inductive coupling.

Based on obtained results, concerning the efficiency of narrowband transmission, it was possible to determine the frequency modes used in BPL-PLC broadband technology for bi-directional voice transmission. Studies of the narrowband transmission also allowed verifying the accuracy of an assumption, that when modeling the impact of cable parameters and frequency on the efficiency of the inductioninductive and mixed capacitive-inductive coupling, it can be determined, accordingly. Confirmation of this can be depicted as a comparison of calculated and measured values of the damping factor $\mathrm{e}^{\alpha}$ versus frequency as shown in Figs. 7 and 8 .

This comparison showed that the assumption for the wave propagation values of all electrical quantities of the cable, in accordance with the geometric structure and cable parameters, type of coupling, and their dependence on frequency, gives reliable results [9].

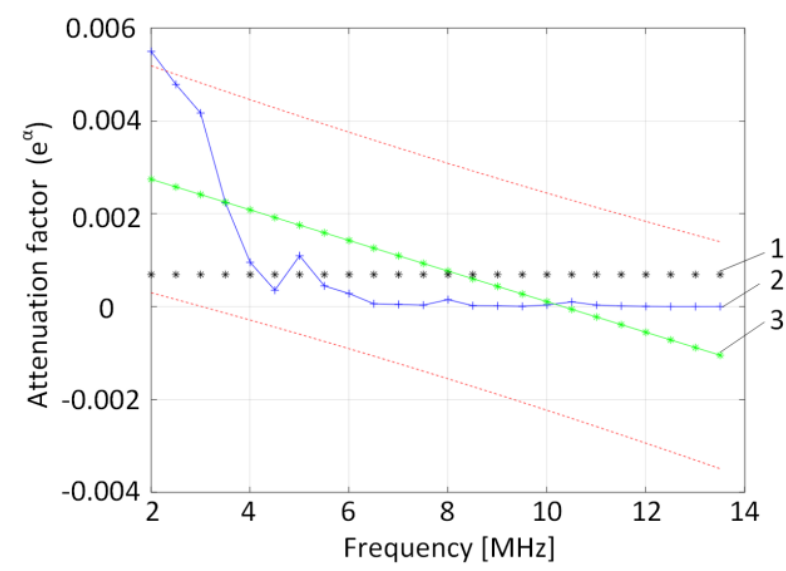

Fig. 7. Attenuation factor $\mathrm{e}^{\alpha}$ versus frequency for induction-inductive coupling: (1) calculated, (2) measured, (3) regression line. 


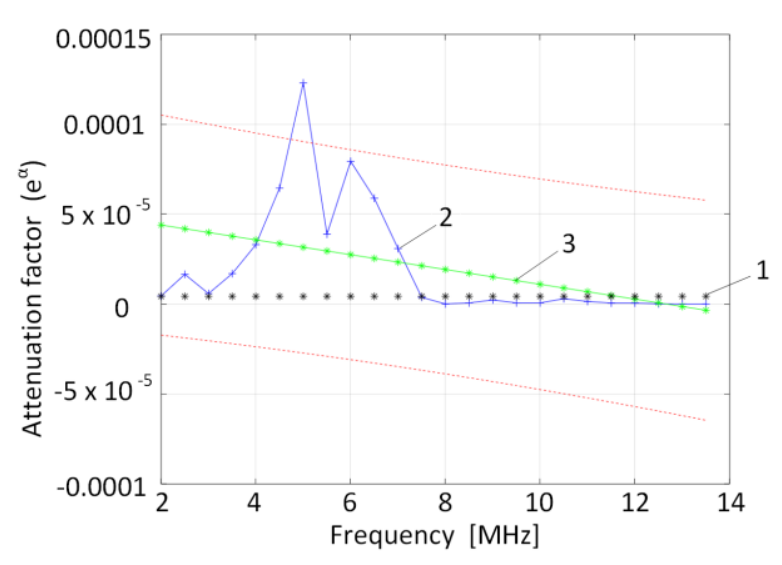

Fig. 8. Attenuation factor $\mathrm{e}^{\alpha}$ versus frequency for capacitive-inductive coupling: (1) calculated, (2) measured, (3) regression line.

They are useful from a practical point of view in order to assess the correct selection of coupling for a given type of a medium voltage mining cable.

\section{BPL Bi-Directional Voice Transmission}

Having specified the highest efficiency of the narrowband transmission for the tested type of medium voltage $6 \mathrm{kV}$ cable within frequency range between $2 \mathrm{MHz}-8 \mathrm{MHz}$, one can choose the appropriate mode. Authors used two different modes marked as MODE $1(3 \mathrm{MHz}-7.5 \mathrm{MHz})$ and MODE $11(2 \mathrm{MHz}-7 \mathrm{MHz})$. In this case, the broadband technology was utilized for bi-directional voice transmission (from A to $B$ and vice versa).

The tests were carried out in an arrangement, as shown in Fig. 9, for both capacitive-inductive and induction-inductive coupling of the BPL-PLC cable.

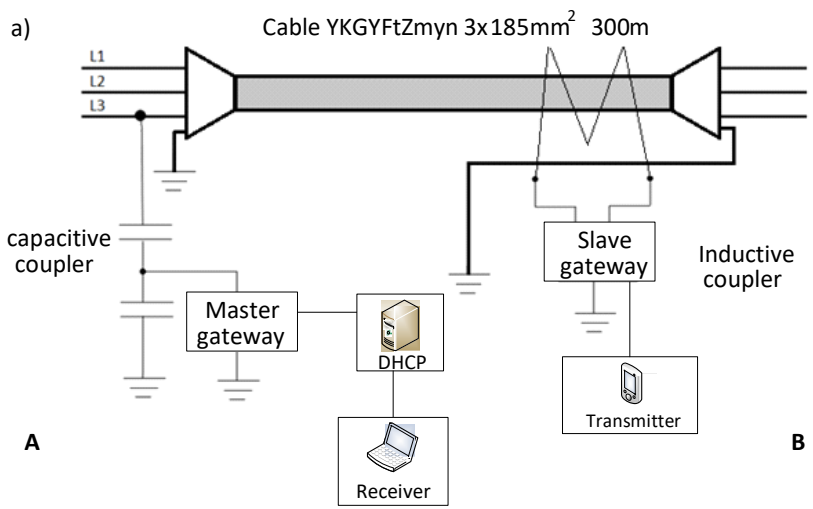

b)

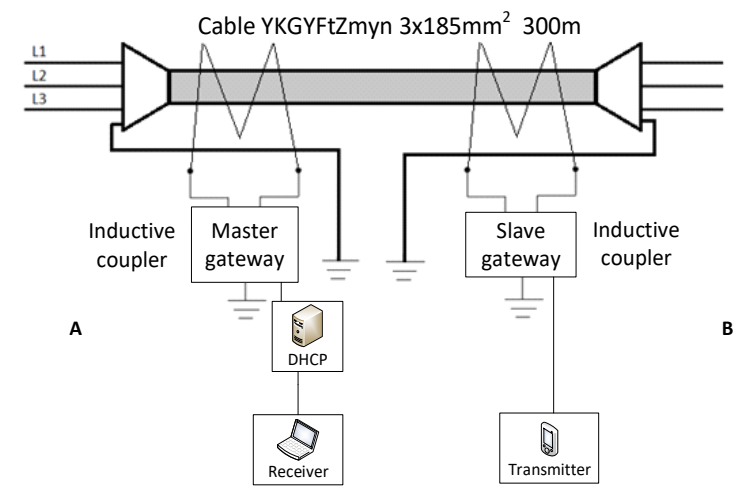

Fig. 9. BPL bi-directional voice transmission measurements: a) capacitiveinductive coupling, b) induction-inductive coupling.

In this case, a set of specially developed digital modules serving as a transmitter and receiver of voice messages were utilized at both ends. The purpose of this research was to determine the minimum bitrate necessary to send intelligible voice messages and not overload the link.

Tests showed that BPL-PLC transmission itself is asymmetrical and its efficiency, resulting in available bitrate, is higher for MODE 11 and for induction-inductive coupling. Detailed results of this investigation are shown in Table I. The value of SNR ranged between $25 \mathrm{~dB}-35 \mathrm{~dB}$, regardless of the type of coupling used.

TABLE I. AVAILABLE BITRATE FOR BPL-PLC TRANSMISSION.
\begin{tabular}{|c|c|c|c|}
\hline Type of Coupling & Direction & Mode & Bitrate [Mbps] \\
\hline \multirow{3}{*}{ Induction-Inductive } & \multirow{2}{*}{ A to B } & 1 & Approximately 23 \\
\cline { 3 - 4 } & & 11 & Approximately 34 \\
\cline { 3 - 4 } & \multirow{2}{*}{ B to A } & 1 & Approximately 18 \\
\cline { 3 - 4 } & & 11 & Approximately 27 \\
\hline \multirow{3}{*}{ Capacitive-Inductive } & \multirow{2}{*}{ A to B } & 1 & Approximately 18 \\
\cline { 3 - 4 } & \multirow{2}{*}{ B to A } & 11 & Approximately 20 \\
\cline { 3 - 4 } & & 11 & Approximately 8 \\
\cline { 3 - 4 } & & & \\
\hline
\end{tabular}

\section{SubJective Evaluation Tests}

The aim of the subjective evaluation test was to confirm and link how do varying parameters of the BPL-PLC wired medium, regarding type of coupling, transmission mode, etc., affect the end user quality. In this case, the term quality itself is referred to as observed difference resulting in perceived speech signal quality degradation. The subjective verification, compared to objective simulation and measurement results, is performed by human individuals.

The subjective assessment was carried out using Beyerdynamic Custom One headphones and involved a group of 15 people aged between 25-35 years old. None of them had hearing disorders. Each individual assessed the quality individually in a 5-step MOS (Mean Opinion Score) ODG (Objective Difference Grade) scale according to [17] and took a training phase before starting the essential study. The ODG scale ranging from -4.0 (very annoying) to 0 (imperceptible) is described in Table II.

TABLE II. OBJECTIVE DIFFERENCE GRADE SCALE.

\begin{tabular}{|c|c|}
\hline Grade & Description \\
\hline 0 & Imperceptible \\
\hline-1.0 & Perceptible, but not annoying \\
\hline-2.0 & Slightly annoying \\
\hline-3.0 & Annoying \\
\hline-4.0 & Very annoying \\
\hline
\end{tabular}

The results of the subjective quality evaluation, including speech samples in British English (EN), German (GE), and Polish (PL), are shown in Figs. 10-12. It should be noted that these results have been averaged for both communication directions, namely, A to B and B to A.

In this case, IC represents induction-inductive coupling, whereas CC represents capacitive-inductive coupling. When statistically processing obtained data, the confidence intervals were set to $5 \%$. Additional information on compression and low bitrate audio coding may be found in [18]-[24]. 


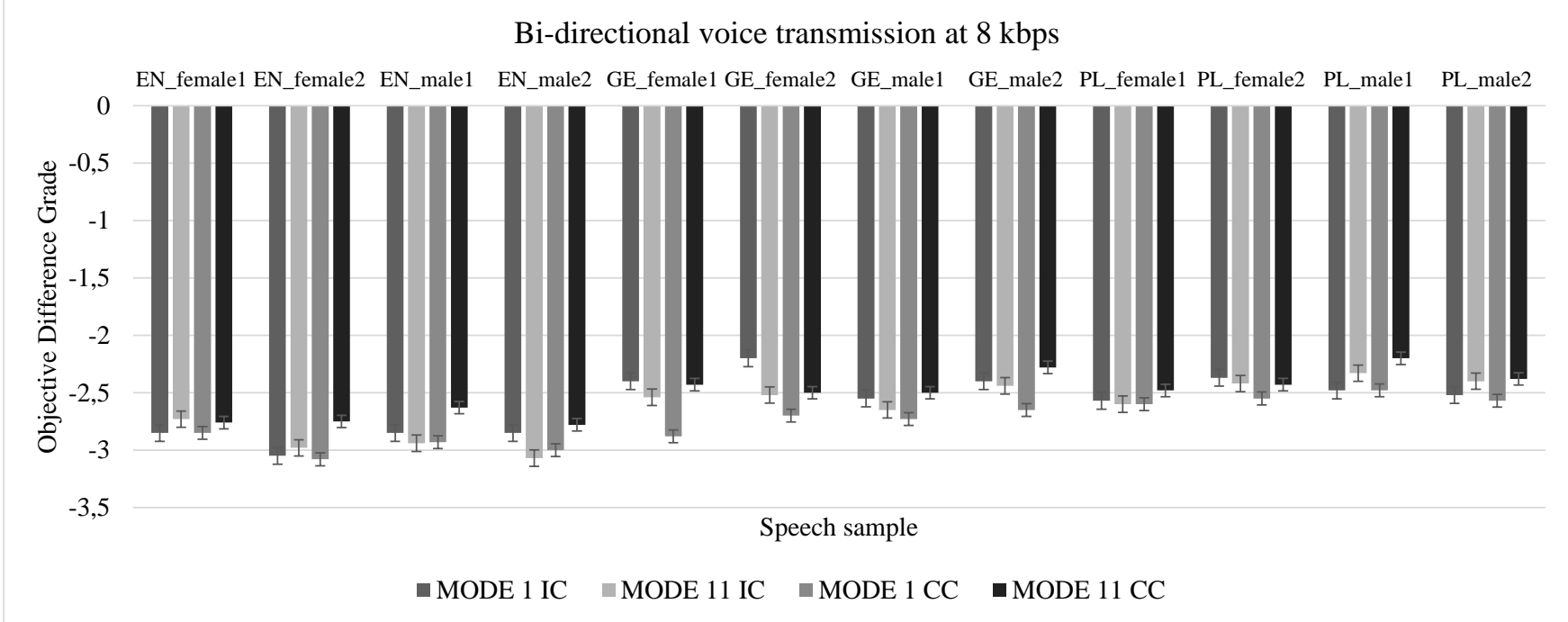

Fig. 10. Quality degradation depending on type of coupling, as well as mode for speech samples processed at $8 \mathrm{kbps}$.

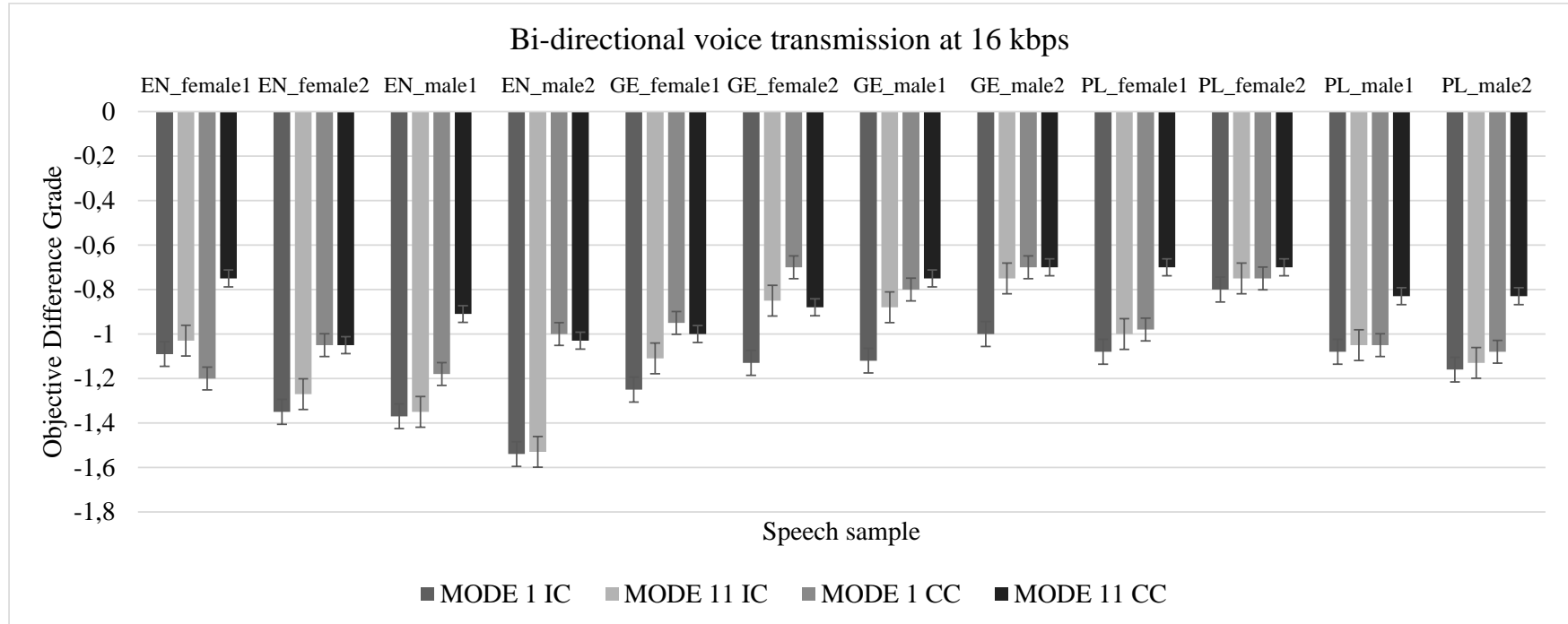

Fig. 11. Quality degradation depending on type of coupling, as well as mode for speech samples processed at $16 \mathrm{kbps}$.

Bi-directional voice transmission at $24 \mathrm{kbps}$

EN_female1 EN_female2 EN_male1 EN_male2 GE_female1 GE_female2 GE_male1 GE_male2 PL_female1 PL_female2 PL_male1 PL_male2

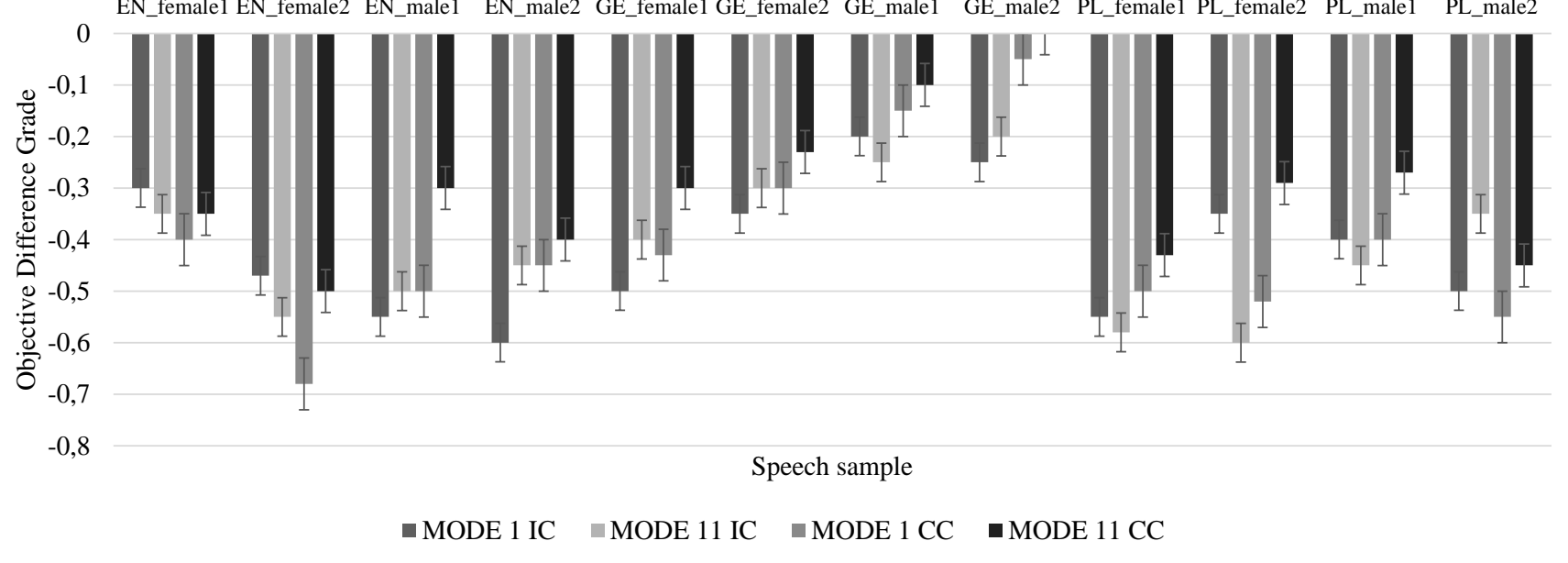

Fig. 12. Quality degradation depending on type of coupling, as well as mode for speech samples processed at $24 \mathrm{kbps}$.

When examining these results, one should take into account that any voice transmission system may be considered as of high-quality whenever it receives a ODG score of -1.0 and above (perceptible, but not annoying). As it is shown, the lowest bitrate equal to $8 \mathrm{kbps}$ proved to be insufficient when it comes to delivering clear and understandable voice messages, which, in this case, were perceived as annoying. On the other hand, the medium bitrate of $16 \mathrm{kbps}$ was ranked evidently better. Nevertheless, not all samples were acceptable. In case of the highest bitrate 
of $24 \mathrm{kbps}$, all voice messages, whether spoken by a male or female lector, were clear and easily understandable.

When it comes to the background of tested individuals, it is worth mentioning that for each one Polish was the mother tongue, whereas both English and German were the second language of choice (without a clear advantage). Moreover, participants pointed out that sentences spoken by a male lector seemed more appealing. In our case, due to the profile of the mining industry, this feedback becomes an important factor. Additional material considering audio signal processing, particularly speech, including psychoacoustic quality measurements in the presence of noise and other interference, may be found in [25]-[29].

\section{CONCLUSIONS}

Broadband BPL-PLC transmission can be effectively used in mine medium voltage cable networks for data transmission, especially voice communication considered as the so-called last chance in case of hazards and/or mine disasters. Such networks are theoretically the most resistant to mechanical damage. Therefore, BPL-PLC combined with speech signal communication, along with battery-powered modems, can be implemented through the screen and cable armor, regardless of its electrical operating conditions.

In order to provide high effectiveness of the BPL-PLC transmission, particularly understandable voice messages, one needs to know how to overcome the influence of environmental interfering EMC factors (both induced and conducted). It also requires appropriate matching of transmitting and receiving devices to the wave impedance of the MV cable. Therefore, the use of effective coupling with the cable is of great importance. It is also necessary to provide a sufficiently high bitrate in order to obtain an appropriate quality of speech signals. It is worth mentioning, that compared to traditional broadcasting and/or streaming services requiring a bitrate of $48 \mathrm{kbps}-64 \mathrm{kbps}$ for speech transmission, the amount of required bandwidth resources can be lowered to approximately $24 \mathrm{kbps}$. Similar remarks were given in a previous study [30].

Described tests carried out for a $6 \mathrm{kV}$ mine cable line of approximately $300 \mathrm{~m}$ long have shown that the effectiveness of bi-directional voice transmission for both rated electrical cable operating conditions, as well as under extreme conditions (lack of supply, shortage and groundage), remains practically the same. When comparing measurement results for unloaded and shorted cables, and for cables loaded with distorted currents, it can be stated that the distortions of current and voltage waveforms (low harmonics $>3^{\text {rd }}$ ) have no effect on the value of noise and the course of their spectrum significant from the point of view considering work efficiency of the BPL-PLC technology. As observed, the maximum noise values did not exceed $-50 \mathrm{dBm}$, and in case of the capacitive coupling, it did not change with frequency.

In case of the tested cable, research on the efficiency of narrowband transmission allowed to select the frequency range of MODE $1(3 \mathrm{MHz}-7.5 \mathrm{MHz})$ and MODE 11 ( $2 \mathrm{MHz}-7 \mathrm{MHz})$ used in BPL-PLC broadband along with voice transmission. Tests showed the asymmetry of the BPL-
PLC medium, but its efficiency (resulting in available bitrate) is slightly higher for MODE 11 and for inductioninductive coupling. In this particular case, these differences are equal to approximately $30 \%$.

When it comes to transmitting voice messages, the analysis showed that speech signals at the lowest bitrate of $8 \mathrm{kbps}$ proved to be insufficient in order to provide clear and unambiguous statements. However, increasing it to $16 \mathrm{kbps}$ does not eliminate this defect completely. Only in case of $24 \mathrm{kbps}$, the voice transmission was clearly understood and acceptable. Similar results were formulated when changing the type of coupling and/or mode.

\section{CONFLICTS OF INTEREST}

The authors declare that they have no conflicts of interest.

\section{REFERENCES}

[1] A. G. Lazaropoulos, "Virtual indicative broadband over power lines topologies for respective subclasses by adjusting channel attenuation statistical distribution parameters of statistical hybrid models (class maps) - Part 3: The case of overhead transmission power grids", Trends in Renewable Energy, vol. 5, no. 3, pp. 282-306, 2019. DOI 10.17737/TRE.2019.5.3.00101.

[2] P. Mlynek, J. Misurec, and M. Koutny, "Modeling and evaluation of power line for smart grid communication", Przegląd Elektrotechniczny, vol. 87, no. 8, pp. 228-232, 2011.

[3] J. Slacik, P. Mlynek, R. Fujdiak, P. Musil, M. Voznak, M. Orgon, and J. Hlavnicka, "Capabilities and visions of broadband power-line in smart grids applications", in Proc. of International Scientific Conference on Electric Power Engineering (EPE 2019), Kouty nad Desnou, 2019, pp. 1-5. DOI: 10.1109/EPE.2019.8777935.

[4] S. H. Mahmood, A. M. Salih, and M. I. Khalil, "Broadband services on power line communication systems: A review", in Proc. of International Conference on Control Systems and Computer Science (CSCS 2019), Bucharest, 2019, pp. 465-470. DOI: 10.1109/CSCS.2019.00085.

[5] H. Meng, S. Chen, Y. L. Guan, C. L. Law, P. L. So, E. Gunawan, and T. T. Lie, "Modeling of transfer characteristics for the broadband power line communication channel", IEEE Transactions on Power Delivery, vol. 19, no. 3, pp. 1057-1064, 2004. DOI: 10.1109/TPWRD.2004.824430.

[6] D. Pyda, M. Habrych, K. Rutecki, and B. Miedzinski, "Analysis of narrow band PLC technology performance in low-voltage network", Elektronika ir Elektrotechnika, vol. 20, no. 5, pp. 61-64, 2014. DOI: 10.5755/J01.EEE.20.5.7101.

[7] G. Debita, P. Falkowski-Gilski, M. Habrych, B. Miedzinski, J. Wandzio, and P. Jedlikowski, "Quality evaluation of voice transmission using BPL communication system in MV mine cable network", Elektronika ir Elektrotechnika, vol. 25, no. 5, pp. 43-46, 2019. DOI: 10.5755/J01.EIE.25.5.24355.

[8] G. Debita, M. Habrych, A. Tomczyk, B. Miedzinski, and J. Wandzio, "Implementing BPL transmission in MV cable network effectively", Elektronika ir Elektrotechnika, vol. 25, no. 1, pp. 59-65, 2019. DOI: 10.5755/J01.EIE.25.1.22737.

[9] G. Debita, M. Habrych, G. Wisniewski, B. Miedzinski, and A. Tomczyk, "Investigation and evaluation of coupling effect of BPL transmission units in MV cable network", in Proc. of International Symposium on Electrical Apparatus and Technologies (SIELA 2018), Bourgas, 2018, pp. 1-4, 2018. DOI: 10.1109/SIELA.2018.8446652.

[10] P. Gilski, "DAB vs DAB + radio broadcasting: A subjective comparative study", Archives of Acoustics, vol. 42, no. 4, pp. 157165, 2017. DOI: 10.1515/AOA-2017-0074.

[11] K. Ulovec and M. Smutny, "Perceived audio quality analysis in digital audio broadcasting plus system based on PEAQ", Radioengineering, vol. 27, no. 1, pp. 342-352, 2018. DOI: 10.13164/RE.2018.0342.

[12] K. Zyka, "The digital audio broadcasting journey from the lab to listeners - The Czech Republic case study", Radioengineering, vol. 28, no. 2, pp. 483-490, 2019. DOI: 10.13164/RE.2019.0483.

[13] A. Dobrucki, S. Brachmanski, and M. Kin, "Objective and subjective evaluation of musical and speech recordings transmitted by DAB+ 
system”, Vibrations in Physical Systems, vol. 30, pp. 1-8, 2019.

[14] Test signals for telecommunication systems, ITU-T Recommendation P.501, 2017.

[15] USB-SA44B spectrum analyzer user manual, Signal Hound, 2019.

[16] AFG-2225 user manual, GW INSTEK, 2019.

[17] General methods for the subjective assessment of sound quality, ITU-R Recommendation BS.1284, 2003.

[18] S. Kandadai and C. D. Creusere, "Scalable audio compression at low bitrates", IEEE Transactions on Audio, Speech, and Language Processing, vol. 16, no. 5, pp. 969-979, 2008. DOI 10.1109/TASL.2008.925881.

[19] E. Ravelli, G. Richard, and L. Daudet, "Union of MDCT bases for audio coding", IEEE Transactions on Audio, Speech, and Language Processing, vol. 16, no. 8, pp. 1361-1372, 2008. DOI: 10.1109/TASL.2008.2004290.

[20] T. Li, S. Rahardja, and S. N. Koh, "Fixed quality layered audio based on scalable lossless coding", IEEE Transactions on Multimedia, vol 11, no. 3, pp. 422-432, 2009. DOI: 10.1109/TMM.2009.2012917.

[21] M. Neuendorf, P. Gournay, M. Multrus, J. Lecomte, B. Bessette, R. Geiger, S. Bayer, G. Fuchs, J. Hilpert, N. Rettelbach, R. Salami, G. Schuller, R. Lefebvre, and B. Grill, "Unified speech and audio coding scheme for high quality at low bitrates", in Proc. of IEEE International Conference on Acoustics, Speech and Signal Processing (ICASSP 2009), Taipei, 2009, pp. 1-4. DOI 10.1109/ICASSP.2009.4959505.

[22] L. Jiang, R. Hu, X. Wang, M. Zhang, and Z. Wang, “AVS2 speech and audio coding scheme for high quality at low bitrates", in Proc. of IEEE International Conference on Multimedia and Expo Workshops (ICMEW 2014), Chengdu, pp. 1-6, 2014. DOI: 10.1109/ICMEW.2014.6890693.

[23] T. Vaillancourt, V. Malenovsky, R. Salami, Z. Liu, L. Miao, J. Gibbs, and M. Jelinek, "Advances in low bitrate time-frequency coding", in Proc. of IEEE International Conference on Acoustics, Speech and
Signal Processing (ICASSP 2015), Brisbane, 2015, pp. 5913-5917. DOI: 10.1109/ICASSP.2015.7179106.

[24] M. Movassagh and P. Kabal, "Scalable audio coding using trellisbased optimized joint entropy coding and quantization", IEEE/ACM Transactions on Audio, Speech, and Language Processing, vol. 24, no. 12 , pp. 2288-2300, 2016. DOI: 10.1109/TASLP.2016.2607339.

[25] J. Kotus, M. Szczodrak, A. Czyzewski, and B. Kostek, "Distributed system for noise threat evaluation based on psychoacoustic measurements", Metrology and Measurement Systems, vol. 19, no. 2 , pp. 219-230, 2012. DOI: 10.2478/V10178-012-0019-6.

[26] C. R. Helmrich, G. Markovic, and B. Edler, "Improved low-delay MDCT-based coding of both stationary and transient audio signals", in Proc. of IEEE International Conference on Acoustic, Speech and Signal Processing (ICASSP 2014), Florence, 2014, pp. 6954-6958. DOI: 10.1109/ICASSP.2014.6854948.

[27] J. Lin, W. Min, Y. Shengyu, G. Ying, and X. Mangan, "Adaptive bandwidth extension of low bitrate compressed audio based on spectral correlation", in Proc. of International Conference on Intelligent Computation Technology and Automation (ICICTA 2015), Nanchang, 2015, pp. 113-117. DOI: 10.1109/ICICTA.2015.37.

[28] B. Kostek and K. Kakol, "Improving the quality of speech in the conditions of noise and interference", Journal of the Acoustical Society of America, vol. 144, no. 3, pp. 1905-1905, 2018. DOI: 10.1121/1.5068349.

[29] Z. Meng, Y. Gaur, J. Li, and Y. Gong, "Speaker adaptation for attention-based end-to-end speech recognition", in Proc. of Annual Conference of the International Speech Communication Association (INTERSPEECH 2019), Graz, 2019, pp. 241-245. DOI: 10.21437/INTERSPEECH.2019-3135.

[30] P. Falkowski-Gilski, "Transmitting alarm information in DAB+ broadcasting system", in Proc. of Signal Processing: Algorithms, Architectures, Arrangements, and Applications (SPA 2018), Poznan, 2018, pp. 217-222. DOI: 10.23919/SPA.2018.8563288. 